\title{
Einige Bemerkungen zur Vogelfauna von Nowaja Semlja.
}

\author{
Von Herman Schalow, Berlin.
}

In der Dansk Ornithologisk Forenings Tidsskrift (10. Jahrg. 1916, III/IV, 145-190) veröffentlichte Tho. L. Schananing vor kurzem eine kleine Arbeit über die von ihm auf Nowaja Semlja gesammelten bzw. beobachteten Vögel, der ich einige Bemerkungen über die Avifauna der genannten arktischen Doppelinsel anfügen möchte.

Nowaja Semlja reicht in ihrer nordsüdlichen Längsrichtung, gegenüber anderen Inselgebieten des östlichen arktischen Meeres, am weitesten nach Süden. Der südlichste Punkt der Insel, Kap Kussow NoB, liegt unter dem $70,32^{n} \mathrm{n}$. Br. Die der Südspitze vorgelagerte, durch die Karastraße von Nowaja Semlja getrennte Insel Waigatsch verbindet sie mit dem Randgürtel des sibirischen Festlandes. Die zuerst von Barents 1596 umfahrene Nordspitze der Insel mit dem Kap Mauritius liegt unter dem $77^{\prime \prime}$ n. Br., d. h. sie liegt nach der Bergeaus'schen Chart of the World (vgl. Ergänz.-Heft 21 der Petermans'schen Mitteilungen 18ii7, 'Taf. 1) uoch siidlicher als die Südspitze von Spitzbergen und die sïdlichsten Inseln des Franz-Joseph-Landes. Mit anderen Worten: Nowaja Semlja reicht im Norden nicht weit in das arktische Meeresgebiet hinein, während sie andererseits weit nach Süden hinabgeht. Diese Lage ist natürlich für die Zusammensetzung der Vogelfauna von wesentlicher Bedeutung. Während die Südgebiete der Doppelinsel einen ansgeprägt flachen Charakter zeigen, mit Sandbänken und einem von niedrigen Riffen umsäumten Strand, steigt weiter nach Norden der Boden in steil aufragenden Bergzügen empor, die dicht an der Küste die Buchten und Randklippen umfassen. Aus beträchtlichen Höhen gehen zahlreiche Bäclie zum Meere. Die bis zu $1000 \mathrm{~m}$ ansteigenden Schroffen sind mit Schnee bedeckt, der sich in breiten, massigen Streifen zum Küstengürtel hinabzieht. Eine mächtige Tonschieferformation baut die Iusel auf. Von vielen Forschern, wie z. B. von KarL Ernst von BaER, wird sie als ein letzter Ausläufer des Uralgebirges betrachtet.

Nach den Beobachtungen PacHTussofrs schwankt die Temperatur zwischen $-23,70^{\circ}$ (März) und $+3,06^{\circ}$ (August). Die Mitteltemperatur liegt bei $-9,45^{\circ}$ Fahrenheit. Von diesen 
Temperaturschwankungen, den dadurch bedingten klimatischen Verhältnissen und von der Bodenbeschäffenheit der Insel ist das Leben der Tier- und Pflanzenwelt, das nur an eine kurze Sommerperiode gebunden erscheint, abhängig. Nạch den Forschungen N. W. Fenuden's (J. Pearson, Beyond Petsora eastward, London 1899, App. A.) können wir für Nowaja Semlja rund 210 Phanerogamen und Kryptogamen annehmen. Während Waigatsch, zwischen $70,25^{\circ}$ und $69,40^{\circ} \mathrm{n}$. Br. gelegen, in seiner Flora noch verschiedene Salix und Betula-Arten, Gramineen, Juncaceen und andere Tundraformen aufweist, treten im Süden von Nowaja Semlja diese Vegetationstypen bereits stark zurück. Weiter im Norden ist das Pflanzenleben auf die Küste und die eis- und schneefreien Täler beschränkt. In lö̈heren Lagen ist die Vegetation sparsam und kümmerlich vertreten. Die Zahl der Arten nimmt nach Norden schnell ab. Während bei einer Breite von $70^{\circ}$ noch 158 Formen auftreten, finden wir bei $75^{\circ}$ nur noch 49 und bei $76,5^{\circ}$ deren 4 . Die im Südosten des Lütkelandes gelegenen Pachtussoff-Inseln besitzen nur 50 Arten. Das nördliche Barentsland ist unerforscht. Seltsamerweise spielen, im Verhältnis zu dem nördlicher gelegenen Spitzbergen, Moose und Flechten eine untergeordnete Rolle. Vielleicht steht diese Erscheinung mit dem fast ausschlieblich anftretenden Schiefergestein in Verbindung und gibt: eine Erklärung für das Fehlen von Schneehühnern.

Es braucht nicht darauf hingewiesen $z u$ werden, daB die Nähe bzw. die fast direkte Verbindung Nowaja Semljas über Waigatsch mit dem sibirischen Festlande für die ornithologischen Ver'bältnisse der Insel von hervorragender Bedeutung sein müssen. Vorkommen, Verbreitung und Zugerscheinungen werden hierdurch in hohem Grade beeinflußt. Auch die im Süden nuch relativ reiche Pflanzenwelt, welche ein, wenn auch nur geringes und kurzes Insektenleben ermöglicht, ist bei der Beurteilung der Ornis der Inseln in Betracht zu ziehen. Von größerer Wichtigkeit vielleicht als diese Faktoren wird sich bei längerem Aufenthalt und eingehenderer ornithologischer Erforschung von Nowaja Semlja, besonders des heute noch unbekannten Barentslandes, aber die Tatsache erweisen, daß um die Spitze der Nordinsel ein warmer Golfstrom herumführt. Diese Entdeckung ist der unter der Führung des Geologen W. A. Russanow stehenden Russischen Polarexpedition gelungen; nach vielen Richtungen hin eine Entdeckung von weitgehendster Bedeutung. Von Wiggrns, NordenskiöLid, NANSEN und anderen Polarforschern ist der Golfstrom stets im Süden von Nowaja Semlja, im Gebiete der Karischen Straße, gesucht worden. Viele Expeditionsschiffe sind infolge der irrigen Annahme schweren Eispressungen und Gefahren ausgesetzt gewesen.

Von der Russanow'schen Polarexpedition sind einige Vögel aus dem höchsten, bis jetzt unbekannten Norden Nowaja Semljas, heimgebracht worden, die aber kein Licht auf die Zusammensetzung der Avifauna des Lïtkelandes werfen. Ich werde anf 
diese kleine Sammlung noch zurückkommen. Nowaja. Semlja bietet hinsichtlich der Biologie der Vögel nınnigfach arktische Probleme. Prarson hat $z$. B. darauf hingewiesen, daß die das genaunte Gebiet bewohnenden Sommer rögel nie zur Brut schreiten, wenn starke Schneefälle, Schneestürme und starke Eispressungen mit niederen Temperaturen eintreten. Einer der wichtigsten Triebe im Leben des Tieres, der Fortpflanzingstrieb, wird für eine Jahresperiode vollkommen ausgeschaltet. Auf die gerade für Nowaja Semlja periodisch eintretenden ungemein schweren Schnee- und Eisverhältnisse ist sicherlich auch die relativ geringe Anzahl von Brutarten zurückzuführen, die für die Insel, trotz der südlichen Lage derselben, bis jetzt erst nachgewiesen worden ist. Vielleicht liegen die Verhältnisse im Norden. in dem der Golfstrom seinen Einfluß geltend machen wird, lierfür günstiger. Geg:enüber dem Spitzbergenarchipel, der um rund $6^{\circ}$ nördlicher liegt. und nach Kownig (Avifauna Spitzbergensis, 1911) 52 Arten mit 29 Brutvögeln nach den heutigen Forschungen anfweist, bleibt Nowaja Semlja in Hinblick auf seine Lage an Artenzihhl stalk zulück. In meinem Werke über die Vögel del Arktis (Jena 1904, S. 276) führte ich 49 Arten mit drei fraglichen auf, von denen 35 als Brutvögel angenommen wurden. Wenig lat sich in den zwölf Jahren nach dem Erscheinen meiner Arbeit geändert. In dem Werke von SERGIUs Alphiraki aus dem Jahre 1904, urspünglich in russischer Sprache veröffentlicht und dann in einer englischen Übersetzung (The Geese of Europe and Asia, London 1905) erschienen, finden sich bemerkenswerte Mitteilungen über die Nowaja Semlja bewohnenden Anseriden. Ich werde hierauf später zurïckkommen.

Sieben Jahre nach meiner Veröffentlichung finden wir von L. A. Moldschanow eine Arbeit in russischer Sprache (Ornithologische Mitteilungen, Moskau, 2. Jahrg. 1911, 109-111): „Einige Worte über die Vögel an der Nordinsel von Nowaja Semlja." Der Genannte begleitete den Geologen W. A. Russanow auf seiner Polarreise und verweilte vier Sommer, vom Jahre 1907 an, auf der arktischen Doppelinsel. Bei der Fahrt Russanow's auf dem „Dmitry Selunsk" um die nördlichste Spitze Nowaja Semljas brachte Moltschasow eine kleine Sammlung von Vögeln, 12 Arten umfassend, zusammen, die sich jetzt im Museum zu Archangelsk befindet. Ich führe die Spezies hier auf, da aus dem höchsten Norden der Insel noch nichts bekannt ist: Colymbus sp., Uria lomvia lomvia (Kolonien bis $76^{\circ} \mathrm{n}$. Br.), Fratercula sp. (Kolonien unter $75^{1} / 2$ "), Alle alle (Kolonien 76"), Stercorarius parasiticus, Larus. glaucus, Pagophila eburnea, Charadrius hiaticula hiaticula (bis zu $75,5^{\circ} \mathrm{n}$. Br.), Tringa maritima (bis zum nördlichsten Punkt der Insel), Melanonyx sp. (bis zu $76^{\prime \prime}$ n. Br. gefunden; an der nördlichsten Spitze keine Spur von Gänsearten), Nyctea nyctea (bis $74^{\circ}$ I. Br., ob nördlicher, unbekannt) und Plectrophenax nivalis nivalis (bis $76^{\circ} \mathrm{n}$. Br. angetroffen). 
Vor kurzem ist die eingangs erwähnte dänische Veröffentlichung von Schannning den Arbeiten, die wir von Baer, Gilletr, von Heuglin, Theel, Markeam, Pearson und Feriden, über die Vogelfauna von Nowaja Semlja besitzen, hinzugefïgt worden. Schannning hat sich rom August 1902 bis zum Juli 1903, vornehmlich zu meteorologischen Studien, an der Ostküste der Insel, im Matotschin-Schar, aufgehalten. Seine Beobachtungen während dieser Zeit umfassen 34 Arten, die sich bis auf zwei bereits in meiner Liste von 1904 finden. Die eine dieser Arten ist Coracias garrulus garrulus L. Ein totes Stück wurde im März. 1903 auf dem Eise gefunden. Es befindet sich im Zoologischen Museum in Christiania. Eine Parallele zu den auf der Bäreninsel gesammelten Exemplaren von Sturnus vulgaris vulgaris L., Turdus merula merula L. und Loxia curvirostra curvirostra L., wie den von Spitzbergen nachgewiesenen Irrlingen von Upupa epops epops L., Corvus cornix cornix L. und Micropus apus apus L. Man sieht wie weit zentral-enropäisch-paläarktische Formen durch widrige Temperaturverhältnisse nach Norden verschlagen werden können.

Die zweite Art, die nach der Schannning'schen Arbeit neu für Nowaja Semlja ist, ist Falco columbarius aesalon 'I'unst., von dessen Vorkommen im östlich arktischen Gebiet nur zwei Fälle vorlagen. Ein Exemplar wurde in der Karasee, südlich. der Weißen Insel, erlegt. Es befindet sich im Museum in Christiania. Das zweite Stück beobachtete NANSEN in der Nähe der SakoliiInsel in der Jugorstraße (Colletr and NANsen, Birds Norw. North. Polar Exp. 1899, 6). Ferner lag eine Beobachtung Pearson's vor, der ein Exemplar am 17. Juli 1897 bei Habarowa, auf dem sibirischen Festlande, der Südspitze von Waigatsch gegenüber, gelegen, beobachtete. Schasnning teilt nun mit, daß er den kleinen Falken auf dem Zuge nach dem Süden am 16. September 1902 und rïckkehrend am 8. Juni 1903 antraf. "Mehrere beobachtete Horste ließen sich jedoch wegen der schwierigen Terrainverhältnisse nicht untersuchen."

Den von Schaanning mitgeteilten eingehenden meteorologischen Aufzeichnungen sind sehr zahlreiche, die Vogelwelt angehende Mitteilungen angefügt, die hinsichtlich biologischarktischer Fragen interessant sind, anf welche an dieser Stelle nur hingewiesen, aber nicht näher eingegangen werden kann. Sie beziehen sich auf Zug- und Brutverhältnisse. In meiner ersten Liste der Vögel von Nowaja Semlja hatte ich bezüglich des Vorkommens drei Arten mit einem Fragezeichen versehen. Es waren dies Larus affinis ReINH., Phalaropus fulicarius und Lagopus lagopus lagopus. Von diesen drei ist inzwischen Phalaropus durch W. S. BRoce sicher gestellt worden. Der Genannte schoß ein Paar dieser Art am 19. Juni 1898 am Kostin Kap, der Südspitze der Moshdoshapsk-Insel, von dem er mit Sicherheit annahm, daß es sich um ein Brutpaar handelte. Das Nest 
konnte nicht gefunden werden. Larus affinis ReINH. wurde, auch von PEarson und FeILden, nur als wahrscheinlich beobachtet, aufgeführt, da keine Belegexemplare gesammelt werden konnten. Vielleicht beziehen sich diese Mitteilungen der genannten Ornithologen auf den aus dem Taimyrgebiet beschriebenen Larus affinis taimyrensis But. (Ornith. Mitt. Moskau 1911, 149). Lagopus lagopus lagopus wird allein auf die Autorität HEoGurs's (Reisen Nordpolarmeer, 3. Teil, 1894, 104), der die Art allerdings mit einem Fragezeichen versieht, für Nowaja Semlja genannt. Unfern der Tschirakinamündung, an der Westküste der südlichen Insel, fand HEUGLIN Federn, die ihrer Textur nach einem Schneehuhn angehören konnten. Hiergegen wendet sich Prarson (Pearson, l. c. 316). „Wir fanden weder auf Waigatsch noch auf Nowaja Semlja irgend eine Spur vom Schneehuhn. Ich glaube, daB der KarataikhafluB [siidwestlich von Habarova] die nördlichste Grenze in diesem Teile der Welt ist." - PEARson ist, 1907 noch einmal auf diesen Gegenstand zurïckgekommen. Fr betonte nachdrücklich (Bull. Brit. Ornith. Club 1907, 77), dab noch nie eine Lagopus sp. für Nowaja Semlja mit Sicherheit nachgewiesen worden sei, und daB OGILvie Grant die genannte arktische Insel auf der im British Museum befindlichen Verbreitungskarte der Schneehühner irrtümlich verzeichnet habe. Moltschanow bemerkt hinsichtlich des Lagopus: „Irgend welche Spuren des Schneehuhns sind, wie allch zu erwarten, nicht gefunden worden."

Auf Grund älterer Beobachtungen werden wiederholt einige Arten in den Arbeiten ïber Nowaja Semlja aufgeführt, deren Vorkommen durch spätere Reisende nicht sichergestellt worden ist. Belegexemplare wurden nicht gesammelt. Es sind dies nach KarL ERNST von Barr (Bull. scient. publ. par l'Acad. imp. de St. Petersbg. T. 3. 1838): Megalestris skua (BRÜNN) und Larus canus canus L.; ferner nach A. MARKHAM (A Polar Reconnaissance): Colymbus immer (BRÜNs) nnd Charadrius apricarius L. Zum Teil sprechen Gründe zoogeographischer Art gegen ein Vorkommen djeser Spezies auf der genannten arktischen Doppelinsel.

Das Kapitel der in der Arktis bzw. auf Nowaja Semlja rorkommenden Gänse ist ein sehr schwieriges und wenig geklärtes. Ich möchte auf dasselbe hier zurückkommen. Im Jahre 1904 führte ich für das in Rede stehende Gebiet auf:

Anser albifrons (Scop.)

Anser fabalis fabalis (Latr.)

Branta bernicla bernicla (L.)

SchasNning verzeichnet in seinen Beobachtungen, wenn auch in anderer Nomenklatur, dieselben Arten. Moutschanow nennt nur eine "Melanonyx sp“. Er spricht die Vermutung aus, daß es sich um M: arvensis handle.

Nun hat Theodor v. Hedglin (J. f. O. 1872, 121) sehr eingehend eine Saatgans, die er auf Nowaja Semlja sammelte, 
beschriebel, welche er als Anser segetum ansprach. Auf Grund dieser Beschreibung hat SEmenow (Rossija II, 1902, 109) eine neue Art aufgestellt: Anser carneirostris (Melanonyx carneirostris Buturlin, Alphékakx. 1. c. 120). Läßt sich diese Art nach Untersuchung von Reihen begründen, so kommt sie also neben Anser fabalis fabalis Latr. (= segetum GM.), der sie zweifellos sehr nahe steht, auf Nowaja Semlja vor. Noch jüngst hat Sminnow junge Vögel der Form, die sich im Petersburger Museum befinden, gesammelt (Alphiraky, l. c. 115). Dieser For'm schließen sich dann Anser fabalis neglectus Suschк. und Anser fabalis arvensis BreHM an. Beide sollen Brutvögel auf Nowaja Semlja sein. Von ersterer befinden sich von Nosilow gesammelte Exemplare im Moskauer Museum. Ferner besitzt das Petersburger Museum ein altes $\sigma^{\circ}$ von Anser finmarchicus Guns., von VASILOw zur Brutzeit auf Nowaja Semlja gesammelt (Alphéraky, l. c. 66). Neben der von mir bereits früher genannten Brutform Branta bernicla bernicla L. soll nach BuTURLIN (Alpherakx, 1. c. Appendix II; 195) noch Branta bernicla glaucogaster Brerm Nowaja Semlja als brütende Form bewohnen. Wir haben mithin, wie ans vorstehendem hervorgeht, auf dem engen Gebiet einer relativ kleinen arktischen Insel vier Anserbzw. Melanonyx- und zwei Branta-Formen als Brutvögel nebeneinander. Es gibt mithin entweder ternär benannte Formen, die sich räumlich nicht vertreten, sondern neben- und miteinander vorkommen, was den herrschenden Ansichten von dem Begriffe der Subspezies diametral widerspricht; oder aber man muß die obigen Formen als binäre Arten betrachten; oder sie sind schließlich nur als Synonyme von Branta bernicla und Anser fabalis aufzufassen.

Nachstehend gebe ich eine Übersicht der bis jetzt für Nowaja Semlja genannten 54 Arten und Formen. Ein Stern bezeichnet die Brutvögel (44).

Ord. Urinatores.

* Uria lomvia lomvia L.

* Fratercula arctica glacialis

* Cepphus mandtii Lichт.

$$
\begin{aligned}
& \text { * Alle alle L. } \\
& \text { * Colymbus adamsi Gray. } \\
& \text { * " } \quad \text { arcticus L. } \\
& \text { * } \quad \text { stellatus Pontopr. }
\end{aligned}
$$

Ord. Longipennes.

\footnotetext{
*Stercorarius pomarinus T TMM. * Larus leucopterus F FBER. 
Ord. Lamellirostres.

* Mergusmergansermerganser $\mathrm{L}$. " serrator L.

Mareca penelope L.

* Clangula hyemalis L.

* Somateria spectabilis L.

* " mollissima mollissi-

* Oidemia nigra nigra L. ma L.

* Anser finmarchicus GunNer.

* " $\quad$ fabalis fabalis Latr.

* " " $"$ carneirostris

SEM.

* " neglectus Suschr.

?* Branta "bernicla bernicla L.

* " fusca fusca L.

? Anser albifrons Scor.

BREHM.

* Cygnus bewickiibewickii $\mathrm{Y}_{\mathbf{A R R} \text {. }}$

Ord. Cursores.

* Arenaria interpres interpres L. * Phalaropus fulicarius L.

* Squatarola squatarola squata- * " lobatus L.

Calidris arenaria L.

rola L.

* Tringa maritima BRÜNN.

* Charadrius morinellus L. * " " alpina alpina L.

* Charadrius hiaticula hiaticula L.

Ord. Raptatores.

Haliaetus albicilla L.

* Falco columbarius aesalon

Hierofalco rusticolus rusticolus

L. * Nyctea nyctea L.

Tunst.

* Falco peregrinus peregrinus

TUNST. (calidus LatH.?)

Ord. Insessores.

Coracias garrulus garrulus $\mathrm{L}$.

Ord. Oscines.

Hirundo rustica rustica L. * Plectrophenax nivalisnivalis L.

* Anthus cervinus PacL.

* Calcarius lapponicus lappo-

* Otocorys alpestris flava GM.

nicus $\mathrm{L}$.

Von den vorgenannten 54 Arten und Formen sind, nach den Mitteilungen Pranson's, die folgenden 11. auf der Nordinsel noch nicht gefunden worden:

Colymbus adamsi.

Tringa minuta minuta.

Tringa alpina alpina.

Phalaropus fulicarius.

Mergus merganser merganser.

Oidemia nigra nigra.
Oidemia fusca fusca.

Cygnus bewickii bewickii.

Haliaetus albicilla.

Calcarius lapponicus lapponicus.

Anthus cervinus. 
Von der Nordinsel, d. h. vom südlichen Lïtkeland allein, ist bis jetzt nur Tringa maritima BRÜNN nachgewiesen.

Schadnntwg, der, wie bemerkt, ein Jahr am Matotschin-Schar beobachtet und gesammelt liat, bezeichnet Nyctea nyctea, Stercorarius pomarinus und longicaudus als periodische, Stercorarius parasiticus und Charadrius morinellus nur als wahrscheinlich brütende Arten. Nur auf dem Zuge wurden von ihm gefunden: Anser albifrons, Branta bernicla bernicla, Rissa tridactyla tridactyla, Pagophila eburnea, Fulmarus glacialis glacialis und Alle alle. Die letztgenannten Arten und Formen sind übrigens, wenn auch zum Teil allerdings nur aus dem Süden der Insel, als Brutyögel von anderen Reisenden bereits nachgewiesen worden. Genauere Forschungen werden diese Verhältnisse wesentlich verschieben und sicherlich auch das Vorkommen weiterer paläarktischen Arten, die wir bereits von Kolguew, Waigatsch, Dolgoi wie von Habarova kennen für Nowaja Semlja, wenigstens für die Fauna der Südinsel, nachweisen. Dahin gehören u. a. Tringa temminckii LEIsL. Machetes pugnax L., Larus argentatus vegae Palmén, Lagopus lagopus lagopus L., eine Leinzeisigform, welche Pearson als Linota linaria aufführt, ferner Archibuteo lagopus lagopus Brưnn., Anthus pratensis L., Motacilla alba alba L., und Saxicola oenanthe oenanthe L. Das Vorkommen des Rauhfußbussards wies Ptiason für Wargatsch zum ersten Male nach. Er fand am 28. Juni 1897 auf einem Felsen einen Horst mit drei Dunenjungen. Er bemerkt hierzu: "This species had not been previously. recorded from either Wargatsch of Novaya Zemlya." Es darf hinzugefügt werden, daß der Nachweis des Vorkommens für die letztgenannte Insel noch zu erbringen ist. 